Abstract 124 Table 1

\begin{tabular}{ll}
\hline Characteristic & Number of patients \\
\hline Age (mean \pm SD) & $65 \pm 10$ \\
Left ventricular ejection fraction (mean \pm standard deviation) & $59 \pm 14 \%$ \\
Male & $48(83 \%)$ \\
Diabetes & $17(29 \%)$ \\
Previous CABG & $13(22 \%)$ \\
Previous percutaneous coronary intervention & $22(38 \%)$ \\
Previous MI & $10(17 \%)$ \\
Hypertension & $38(66 \%)$ \\
\hline
\end{tabular}

\section{ASSESSING PATIENT BENEFIT FROM THE REVASCULARISATION OF CHRONICALLY OCCLUDED CORONARY ARTERIES BY ADVANCED CARDIOVASCULAR MRI TECHNIQUES}

doi:10.1136/heartjnl-2011-300198.125

${ }^{1} \mathrm{~N} J$ Artis, ${ }^{2} \mathrm{~A}$ Crean, ${ }^{1} \mathrm{~A}$ Zaman, ${ }^{1} \mathrm{~S}$ Sorbron, ${ }^{1} \mathrm{~A} N \mathrm{~N}$ Mather, ${ }^{1} \mathrm{~S} \mathrm{G}$ Ball, ${ }^{1} \mathrm{~S}$ Plein, ${ }^{1} \mathrm{~J}$ P Greenwood. ' University of Leeds, Leeds, UK; ${ }^{2}$ Toronto General Hospital, Toronto, Canada

Background Cardiovascular magnetic resonance (CMR) imaging can provide an array of information about cardiac function and anatomy. The utility of CMR in the setting of coronary artery chronic total occlusion (CTO) has not been fully investigated. We set out to examine the ability of CMR to show regional improvements in left ventricular (LV) function and perfusion and to investigate if any features were able to predict those that benefit from revascularisation. Methods Twenty-seven patients with single vessel CTO were recruited from clinical waiting lists and underwent a comprehensive CMR assessment prior to and 6 months following attempted CTO revascularisation. A multi-parametric CMR protocol was performed which included cine imaging to assess regional wall thickness/ thickening and global LV function, rest and adenosine stress perfusion imaging (Fermi model), low dose dobutamine stress to assess inotropic reserve, and late gadolinium enhancement (LGE) imaging to determine scar location and extent. Using the AHA 16 segment model only segments supplied by the CTO artery were studied for functional improvement. Data are presented as mean (SD).

Results Procedural success in terms of revascularisation of the occluded artery was achieved in 23 of the 27 patients (85\%, 20 with

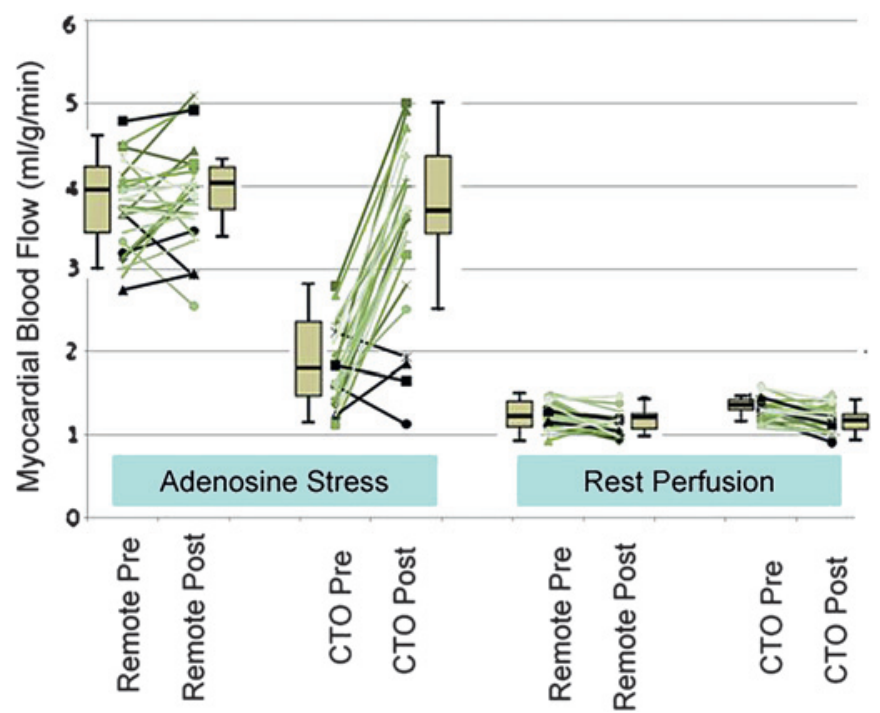

Abstract 125 Figure 1 Changes observed in the CTO and a remote territory following attempted revascularisation. (non-revascularisedblack lines). Adenosine stress increases perfusion only in the CTO territory with no change in resting perfusion.
$\mathrm{PCI}$ and 3 with $\mathrm{CABG}$ ). In those with successful revascularisation by PCI LV volumes reduced (EDV 185 (54) vs 174 (50) p<0.05; ESV $85(60)$ vs $77(58) \mathrm{p}<0.001)$ and the left ventricular ejection fraction improved (56.5(12)\% vs 58.9(12)\% p=0.01). During adenosine stress imaging there was a significant improvement in absolute myocardial blood flow in the revascularised segments (from $1.87(0.51)$ to 3.77 $(0.67) \mathrm{ml} / \mathrm{g} / \mathrm{min} \mathrm{p}<0.001$ ) but not in the remote regions (from 3.76 (0.52) to $3.95(0.58) \mathrm{ml} / \mathrm{g} / \mathrm{min} \mathrm{p}=\mathrm{ns})$. LGE was only present in 25 $(20 \%)$ revascularised segments. In these segments there was a strong inverse correlation between the extent of scar and improvement in segmental systolic thickening $(\mathrm{r}=-0.736, \mathrm{p}<0.001)$. There was a weaker association between the segmental response to low dose dobutamine and the degree of functional improvement following successful revascularisation (Pearson $\mathrm{r}=0.249, \mathrm{p}<0.01$ ).

Conclusion Following revascularisation of $\mathrm{CTO}$, myocardial perfusion increases and both regional and global systolic function improves. While the majority of subjects in this study had no scar on LGE imaging, when segments are scarred there is a negative correlation with improvement in regional systolic thickening.

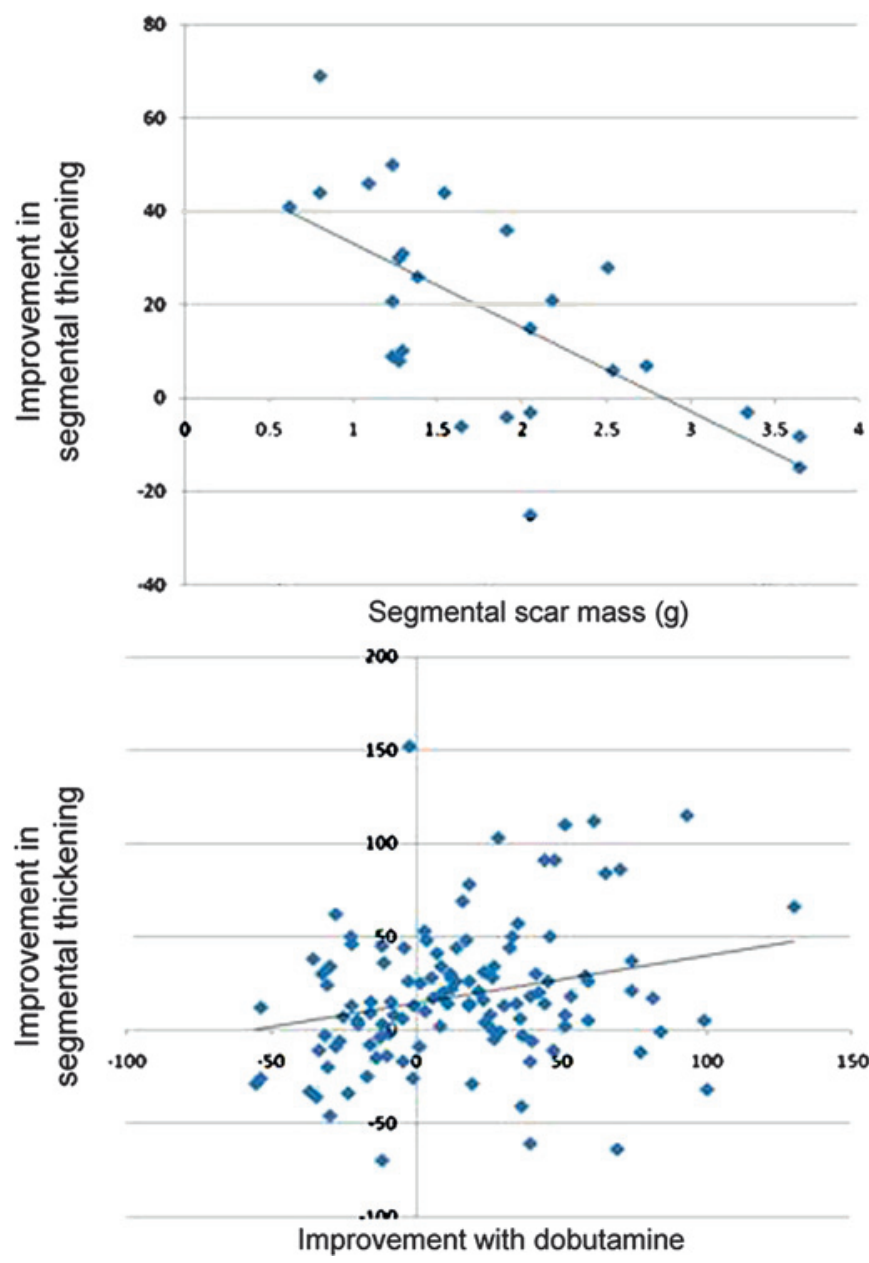

Abstract 125 Figure 2 Relationship of improvement in segmental systolic thickening against segmental scar (top panel) and change in thickening with low dose dobutamine (bottom panel).

\section{THE IMPACT OF NICE GUIDELINES FOR THE INVESTIGATION OF CHEST PAIN ON OUTPATIENT CARDIOLOGY SERVICES IN THE UK}

doi:10.1136/heartjnl-2011-300198.126

${ }^{1} \mathrm{C}$ Patterson, ${ }^{2} \mathrm{E}$ Nicol, ${ }^{3} \mathrm{~L}$ Bryan, ${ }^{4} \mathrm{~T}$ Woodcock, ${ }^{1} \mathrm{~S}$ Padley, ${ }^{1} \mathrm{D}$ Bell. ${ }^{1} / \mathrm{mperial}$ College, London, UK; ${ }^{2}$ Royal Brompton Hospital, London, UK; ${ }^{3}$ Chelsea and Westminster Hospital, London, UK; ${ }^{4}$ NIHR CLAHRC for Northwest London, London, UK 
Introduction The National Institute for Health and Clinical Excellence (NICE) have released guidelines for the investigation of chest pain of recent onset (1). There is concern that the guidelines will increase the burden on cardiac imaging, requiring service reconfiguration and investment $(2,3)$. This study was performed to assess the impact of the guidelines on outpatient cardiology services in the UK.

Methods 595 consecutive patients attending chest pain clinics at two hospitals over six months preceding release of the NICE guidelines (51\% male; median age 55 yrs (range 22-94 yrs)) were risk stratified using NICE criteria. Preliminary cardiac investigations recommended by NICE were compared with existing clinical practice and the relative costs calculated.

Results NICE would have recommended 443 patients (74\%) for discharge without cardiac investigation, 10 (2\%) for cardiac computed tomography (CCT), 69 (12\%) for functional cardiac imaging and $73(12 \%)$ for invasive coronary angiography (ICA). Relative to existing practice there would have been a trend towards reduced functional cardiac imaging $(-24 \% ; p=0.06)$ and increased CCT $(+43 \% ; p=0.436)$ but a significant increase in ICA $(+508 \%$; $\mathrm{p}<0.001)$. The cost of investigations recommended by NICE would have been $£ 15881$ greater than existing practice.

Conclusions This study suggests implementation of the NICE guidelines will require investment in cardiology services, particularly ICA. It will be necessary to establish and maintain CCT for relatively few patients; also to establish and maintain functional cardiac imaging even though referrals are likely to decline. Individual hospitals should assess their local populations prior to service reconfiguration.

Abstract 126 Table 1 Preliminary cardiac investigations undertaken (pre-NICE) compared with those recommended by NICE $(\mathrm{N}=595)$

\begin{tabular}{lccl}
\hline & Pre-NICE & NICE & $\%$ change \\
\hline No investigation & 33 & 443 & $+1242 \%(p<0.001)$ \\
Cardiac CT & 7 & 10 & $+43 \%(p 0.436)$ \\
Functional cardiac assessment & 91 & 69 & $-24 \%(p 0.06)$ \\
Invasive angiography & 12 & 73 & $+508(\mathrm{p}<0.001)$ \\
\hline
\end{tabular}

\section{7}

\section{TIMING OF CARDIOVASCULAR MRI AFTER ACUTE MYOCARDIAL INFARCTION: EFFECT ON ESTIMATES OF INFARCT CHARACTERISTICS AND PREDICTION OF LATE VENTRICULAR REMODELLING}

doi:10.1136/heartjnl-2011-300198.127

A N Mather, T A Fairbairn, N J Artis, J P Greenwood, S Plein. University of Leeds, Leeds, UK

Background The pathophysiological remodelling processes associated with acute myocardial infarction (AMI) evolve over time and the optimal acute imaging time point to predict medium-term surrogates for outcome has not been established. This study aimed to define the evolution of infarct characteristics by cardiovascular magnetic resonance (CMR), and to assess whether CMR data acquired at "day 2" or at " 1 week" post-AMI are stronger predictors of infarct size and left ventricular (LV) function measured at 3 months. Methods Fifty-seven patients were recruited with first presentation ST elevation AMI treated successfully with primary percutaneous coronary intervention. Cine, T2- weighted and late gadolinium enhancement CMR imaging were performed at days 2, 7, 30 and 90 after index presentation.

Results Infarct size and extent of myocardial oedema decreased significantly between "day 2" and "1 week" (mean \%LV-scar (SD) 27.2 (13.9) vs 21.6 (14.1), p<0.001 and \%LV-AAR (Area At Risk) (SD), 37.9 (15.2) vs 32.3 (14.3), $\mathrm{p}=0.003$ ). These changes were accompanied by a significant improvement in LV ejection fraction (\%LVEF (SD), 41.7 (9.6) vs 44.6 (10.1), p<0.001). CMR data acquired at "1 week" were better predictors of LVEF and infarct size at "3 months" than data collected at "day 2".

Conclusions The extent of myocardial oedema and infarct size decrease significantly during the first week after reperfusion for AMI and these changes are associated with a significant improvement in LVEF over the same interval. These findings have implications for the timing of CMR studies in the early post-infarct period. We found that the percentage myocardial salvage index did not change significantly between "day 2" and "1 week". Therefore, accurate assessment of the efficacy of reperfusion therapy can be made up to one week after revascularization. In addition, CMR data acquired at "1 week" were better predictors of CMR endpoints measured at "3 months". Thus, we conclude that the optimal time point to image patients post-reperfusion therapy for AMI is at 1 week.

\section{BRIGHT BLOOD T2 WEIGHTED MRI HAS HIGHER DIAGNOSTIC PRECISION AND ACCURACY THAN DARK BLOOD STIR MRI FOR ASSESSMENT OF THE ISCHAEMIC AREA-AT-RISK AND MYOCARDIAL SALVAGE IN ACUTE MYOCARDIAL INFARCTION}

doi:10.1136/heartjnl-2011-300198.128

${ }^{1} \mathrm{~A}$ R Payne, ${ }^{1} \mathrm{M}$ Casey, ${ }^{1} \mathrm{~J}$ McClure, ${ }^{2} \mathrm{R}$ McGeoch, ${ }^{2} \mathrm{~A}$ Murphy, ${ }^{2} \mathrm{R}$ Woodward, ${ }^{2} \mathrm{~A}$ Saul, ${ }^{2} \mathrm{~J}$ Gilchrist, ${ }^{2} \mathrm{C}$ Clark, ${ }^{2} \mathrm{~K}$ G Oldroyd, ${ }^{1} \mathrm{~N}$ Tzemos, ${ }^{1} \mathrm{C}$ Berry. ${ }^{1}$ University of Glasgow, Glasgow, UK; ${ }^{2}$ Golden Jubilee National Hospital, Glasgow, UK

Background T2-weighted MRI reveals myocardial oedema and enables estimation of the ischaemic area-at-risk and myocardial salvage in patients with acute myocardial infarction (MI). We compared the diagnostic accuracy of a new bright blood T2-weighted with a standard black blood T2-weighted MRI in patients with acute MI.

Methods A breath hold bright blood T2-weighted ACUTE pulse sequence with normalisation for coil sensitivity and a breath hold T2 dark blood short $\tau$ inversion recovery (STIR) sequence were used to depict the area-at-risk in 54 consecutive acute MI patients. Infarct size was measured on gadolinium late contrast enhancement images. Results Compared with dark blood T2-weighted MRI, consensus agreements between independent observers for identification of myocardial oedema were higher with bright blood T2 -weighted MRI when evaluated per patient $(p<0.001)$ and per segment of left ventricle $(p<0.001)$. Compared to bright blood T2-weighted MRI, dark blood T2-weighted MRI under-estimated the area-at-risk compared to infarct size $(\mathrm{p}<0.001)$. The $95 \%$ limits of agreement for inter-observer agreements for the ischaemic area-at-risk and myocardial salvage were wider with dark blood T2-weighted MRI than with bright blood T2-weighted MRI. Bright blood enabled more accurate identification of the culprit coronary artery with correct identification in $94 \%$ of cases compared to $61 \%$ for dark blood ( $p<0.001$ ).

Conclusion Bright blood T2-weighted MRI has higher diagnostic accuracy than dark blood T2-weighted MRI. Additionally, dark blood T2-weighted MRI may underestimate area-at-risk and myocardial salvage.

\section{MYOCARDIAL SALVAGE DURING PRIMARY PCI CAN BE PREDICTED IN THE CATH LAB}

doi:10.1136/heartjnl-2011-300198.129

${ }^{1} \mathrm{~A}$ R Payne, ${ }^{1} \mathrm{C}$ Berry, ${ }^{1} \mathrm{O}$ Doolin, ${ }^{2} \mathrm{M}$ B McEntegart, ${ }^{2} \mathrm{R}$ Woodward, ${ }^{2} \mathrm{~A}$ Saul, ${ }^{2} \mathrm{~S} D$ Robb, ${ }^{2} \mathrm{M}$ C Petrie, ${ }^{1}$ I Ford, ${ }^{2} \mathrm{~K}$ G Oldroyd. ${ }^{1}$ University of Glasgow, Glasgow, UK; ${ }^{2}$ Golden Jubilee National Hospital, Glasgow, UK

Objectives This study investigated the relationship between the index of microcirculatory resistance (IMR) and myocardial salvage as determined by $\mathrm{T} 2$-weighted and contrast-enhanced cardiac magnetic resonance (CMR) imaging in patients undergoing primary percutaneous coronary intervention (pPCI) for ST elevation myocardial infarction (STEMI). 